$\underline{\text { Research article }}$

\title{
A descriptive analysis of clinico-demographic features and microbiological results of patients with suspected typhoid fever in four large hospitals of Bhutan
}

\author{
T Tshokey ${ }^{1}, \mathrm{~N}$ Tshering ${ }^{1}, \mathrm{~K}$ Wangchuk ${ }^{1}$, R Sharma ${ }^{1}$, A Mongar $^{1}$, T Dorji ${ }^{2}, \mathrm{~S} \mathrm{Wangchuk}^{3}$, \\ D Damcho ${ }^{4}$, K Wangdi ${ }^{5}$
}

Sri Lankan Journal of Infectious Diseases 2016 Vol.6 (2):106-113

DOI: http://dx.doi.org/10.4038/sljid.v6i2.8117

\begin{abstract}
Introduction: Bhutan reports about 2000 typhoid fever cases annually. We aimed at understanding the clinico-demographic features and microbiological results of patients with suspected typhoid fever.

Methods: A yearlong (2012) study of typhoid fever suspects was conducted to describe and analyse associations of demographic and clinical features with laboratory findings.

Results: A total of 457 patients were enrolled. Most patients were from Phuntsholing General Hospital $(\mathrm{n}=181 ; 39.6 \%)$ followed by Jigme Dorji Wangchuck National Referral Hospital $(\mathrm{n}=170 ; 37.2 \%)$, Eastern Regional Referral Hospital $(\mathrm{n}=56 ; 12.3 \%)$ and Central Regional Referral Hospital $(n=50 ; 10.9 \%)$. Fever $(n=420 ; 91.9 \%)$ and headache $(n=397 ; 86.9 \%)$ were the commonest symptoms reported by the patients. Only $30 \%(n=137)$ and $11.2 \%(n=51)$ had diarrhoea and constipation respectively. Mean duration of illness was 11.2 days. Among the 457 Widal tests performed, $76.1 \%(n=348)$ were negative, $12.3 \%(n=56)$ positive for $\mathrm{O}$ antigen, $8.5 \%(\mathrm{n}=39)$ for $\mathrm{H}$ antigen and 3.1\% $(\mathrm{n}=14)$ for both. Only 2 of the $109(1.8 \%)$ patients with a positive Widal test had a positive blood culture. Widal test showed a sensitivity of $33.3 \%$ and specificity of $76.3 \%$. There was no association of any symptoms to antibody titres. There were three peaks when suspected cases reported to hospitals. More than 97\% $(\mathrm{n}=447)$ of blood were sterile and Salmonella Typhi was isolated only in $1.3 \%(\mathrm{n}=6)$. Only one isolate showed resistance to amoxicillin and nalidixic acid.
\end{abstract}

Conclusion: Typhoid fever was not being diagnosed satisfactorily but over-diagnosed and treated clinically. Widal test should be replaced by more sensitive and specific tests or used cautiously with well-defined cut-off titres.

Key words: Bhutan, Salmonella Typhi, Typhoid fever, Widal test

\footnotetext{
${ }^{1}$ Microbiology unit, Department of Laboratory Services, JDW National Referral Hospital, Thimphu

${ }^{2}$ Microbiology unit, Phuntsholing General Hospital, Chukha

${ }^{3}$ Microbiology unit, Central Regional Referral Hospital, Gelephu

${ }^{4}$ Microbiology unit, Eastern Regional Referral Hospital, Mongar

${ }^{5}$ Phuntsholing General Hospital, Chukha

Address for correspondence: Dr. Tshokey Clinical Microbiologist, Microbiology Unit, Department of Laboratory Services, Jigme Dorji Wangchuck National Referral Hospital, Thimphu, Bhutan

Email: doc_tshokey@yahoo.com; Phone: +975-2-324566
} 


\section{Introduction}

Typhoid fever is a foodborne systemic infection caused by Salmonella enterica serotype Typhi. It is endemic in many Asian and African countries. In a review, Crump and colleagues (2004) stated that typhoid fever resulted in over 21 million illnesses and 216,510 deaths in the year 2000 globally. ${ }^{1}$ The same review identified south-central and south-east Asia as high incidence locations of typhoid fever (>100/100,000 cases/year), the rest of Asia, Africa, Latin America and the Caribbean, and Oceania (excluding Australia and New Zealand) as medium incidence (10-100/100,000 cases/year), and Europe, North America, and the rest of the developed world as low incidence of typhoid fever $\left(<10 / 100,000\right.$ cases/year). ${ }^{1}$ In a multicentered study involving five Asian countries ${ }^{2}$, the annual typhoid incidence among the 5-15 years age group ranged from 24, 29, 180, 413 and 494 cases per 100,000 person years for study sites in Vietnam, China, Indonesia, Pakistan and India respectively. This study also showed that up to $23 \%$ of the isolates were resistant to commonly used antibiotics (chloramphenicol, ampicillin and trimethoprim-sulfamethoxazole). Typhoid fever is also an infection of high concern for travelers due to high travel associated infections as reported by Steinberg and colleagues in $2004 .^{3}$

Typhoid fever is probably endemic in Bhutan similar to other neighboring countries but no formal research has been carried out to date. On an average, about 2000 cases of typhoid fever were reported annually in Bhutan since $2006 .{ }^{4}$ These annual national reports mostly include clinical cases and laboratory diagnosis based on the Widal test since blood culture is available only in four hospitals in the country. Routine blood cultures in the hospitals have occasionally grown Salmonella Typhi and Salmonella Paratyphi. In 2010, the Jigme Dorji Wangchuck National Referral Hospital (JWNRH), Thimphu and the Eastern Regional Referral Hospital (ERRH), Mongar isolated eight and three $S$. Typhi respectively from routine blood cultures. In the same year, the JDWNRH performed 5,365 Widal tests and the ERRH and Central Regional Referral Hospital (CRRH), Gelephu, performed 615 and 942 Widal tests respectively. Despite its low sensitivity and specificity and being obsolete in many countries, the Widal test is widely used in all hospitals in Bhutan. In fact, it is one of the most available tests for infectious diseases in the country. However, in the absence of an established epidemiology and baseline titer, the antibody titer of 1:80 in a single serum sample to either or both the $\mathrm{O}$ and $\mathrm{H}$ antigens is currently regarded as positive in the Bhutanese setting but the practice is not uniform. Repeated tests with positive titers during subsequent visits in the same patient followed by antibiotic treatment seem to be common in daily clinical practice (personal communication with clinicians).

The aim of this study was to identify the clinico-demographic features and microbiological test results of patients with suspected typhoid fever presenting in the four large hospitals of Bhutan.

\section{Methods}

This is a descriptive analysis of data obtained from a yearlong pilot study of typhoid fever suspected patients between January-December 2012. The study was conducted in the three referral hospitals (JDWNRH, ERRH, CRRH) and Phuntsholing General Hospital (PGH), the only four hospitals with microbiological culture facilities in Bhutan. This was carried out as a routine public health activity of the Department of Public Health (DoPH), Ministry of Health $(\mathrm{MoH})$ using these microbiology laboratories. 
A study protocol was developed involving the relevant people. Laboratory staff and clinicians in the four hospitals were briefed of the study before commencement. All patients (any age and sex) presenting to the four hospitals with febrile illness, suspected of typhoid fever by the examining physicians after proper history taking and clinical exclusion of focal/systemic causes of fever whose serum sample sent to the laboratory for Widal test were included in the study. No chronically ill patients and those with obvious signs and symptoms or clinical finding of other causes of fever were included. Recruitment of patients was purely based on clinical diagnosis of typhoid fever suspects by clinicians. Information on common clinical signs and symptoms and duration of illness was recorded by the examining physicians for each patient. All participants submitted blood for Widal test (single acute serum sample) and culture.

A quantitative rapid slide test method of Widal test for Salmonella antigens from Reckon Diagnostics P. Ltd, India, was used in all hospitals as per manufacturer's instructions. An antibody tire of 1:80 against one or both of the $\mathrm{O}$ and $\mathrm{H}$ antigens was considered positive. Blood culture was processed in BacT/Alert automated aerobic culture systems from Biomerieux, USA, and any positive growth processed following routine microbiological procedures. Isolates were identified according to growth characters, Gram stain appearances, biochemical tests and serotyping using Salmonella serotype agglutination kits. Antibiotic susceptibility testing (ABST) was carried out according to the Clinical and Laboratory Standards Institute (CLSI) guideline. The laboratory procedures in the four participating hospitals were standardized through the supply of same reagents and test kits and prior briefing and coordination meetings of laboratory staffs before the study commenced.

\section{Statistical analysis}

Data analysis was carried out using the statistical package STATA 13 (Stata Corporation, College Station, Texas, USA). Descriptive statistics were carried out by providing the number and percentage of each of the symptoms and Widal test. Case incidence was calculated using blood culture positives and Widal test positives separately. Sensitivity, specificity, negative and positive predictive values for Widal test was calculated considering blood culture positives as the true indicator of typhoid fever.

\section{Ethical approval}

Since this was carried out as a routine public health activity of the Department of Public Health, Ministry of Health, ethical approval was not required by local guidelines. In addition, no personally identifiable information on individual patients was collected.

\section{Results}

A total of 457 patients were enrolled during the study period. The highest cases were reported by PGH with 39.6\% (181) followed by JDWNRH with 37.2\% (170) and CRRH recorded the least at $10.9 \%$ (50). There was almost equal number of male and female patients. Overall average age of patients was 31.6 years (95\% CI 30.2, 33.1). CRRH recorded the younger patients (mean age 27.8 years) while JDWNRH recorded older patients (mean age 34.4 years) (Table 1).

The study recorded five common symptoms; fever, headache, body ache, diarrhoea and constipation. The commonest symptoms recorded were fever and headache with about $92 \%$ $(n=420)$ complaining of fever and $87 \%(n=387)$ complaining of headache. Only $30 \%(n=137)$ 
and $11.2 \%$ (51) presented with diarrhoea and constipation respectively. Mean duration of illness before medical consultation was 11.2 days but CRRH did not record the duration of illness (Table 2).

Table 1: Gender and mean age by different hospitals

\begin{tabular}{|c|c|c|c|c|}
\hline \multirow[b]{2}{*}{ Hospital } & \multirow[b]{2}{*}{ Cases (\%) } & \multicolumn{2}{|c|}{ Gender (\%) } & \multirow[b]{2}{*}{ Mean age $(95 \%$ CI $)$} \\
\hline & & Female & Male & \\
\hline CRRH, Gelephu & $50(10.9)$ & $21(42.0)$ & $29(58.0)$ & $27.8(22.9,32.7)$ \\
\hline ERRH, Mongar & $56(12.3)$ & $33(41.1)$ & $23(58.9)$ & $29.8(26.2,33.5)$ \\
\hline PGH, Phuentsholing & $181(39.6)$ & $87(48.1)$ & $94(51.9)$ & $30.7(28.6,32.9)$ \\
\hline JDWNRH, Thimphu & $170(37.2)$ & $88(53.3)$ & $78(46.7)$ & $34.4(32.1,36.8)$ \\
\hline Total & $457(100.0)$ & $229(50.6)$ & $223(49.4)$ & $31.6(30.2,33.1)$ \\
\hline
\end{tabular}

Table 2: Symptoms reported by different hospitals

\begin{tabular}{|c|c|c|c|c|c|c|c|c|c|c|}
\hline \multirow{2}{*}{ Hospital } & \multicolumn{2}{|c|}{ Fever $(\%)$} & \multicolumn{2}{|c|}{ Headache (\%) } & \multicolumn{2}{|c|}{ Body ache (\%) } & \multicolumn{2}{|c|}{ Diarrhoea (\%) } & \multicolumn{2}{|c|}{ Constipation (\%) } \\
\hline & Yes & No & Yes & No & Yes & No & Yes & No & Yes & No \\
\hline CRRH & $\begin{array}{c}48 \\
(96.0)\end{array}$ & $\begin{array}{c}2 \\
(4.0)\end{array}$ & $\begin{array}{c}45 \\
(90.0)\end{array}$ & $\begin{array}{c}5 \\
(10.0)\end{array}$ & $\begin{array}{c}47 \\
(94.0)\end{array}$ & $3(6.0)$ & $\begin{array}{c}20 \\
(40.0)\end{array}$ & $\begin{array}{c}30 \\
(60.0)\end{array}$ & $\begin{array}{c}9 \\
(18.0)\end{array}$ & $\begin{array}{c}41 \\
(82.0)\end{array}$ \\
\hline ERRH & $\begin{array}{c}56 \\
(100.0) \\
\end{array}$ & $\begin{array}{c}0 \\
(0.0) \\
\end{array}$ & $\begin{array}{c}45 \\
(80.4) \\
\end{array}$ & $\begin{array}{c}11 \\
(19.6) \\
\end{array}$ & $\begin{array}{c}41 \\
(73.2) \\
\end{array}$ & $\begin{array}{c}15 \\
(26.8) \\
\end{array}$ & $\begin{array}{c}31 \\
(55.4) \\
\end{array}$ & $\begin{array}{c}25 \\
(44.6) \\
\end{array}$ & $\begin{array}{c}2 \\
(3.6) \\
\end{array}$ & $\begin{array}{c}54 \\
(96.4) \\
\end{array}$ \\
\hline PGH & $\begin{array}{c}180 \\
(99.5)\end{array}$ & $1(0.5)$ & $\begin{array}{c}166 \\
(91.7) \\
\end{array}$ & $\begin{array}{c}15 \\
(8.3) \\
\end{array}$ & $\begin{array}{c}150 \\
(82.9)\end{array}$ & $\begin{array}{c}31 \\
(17.1)\end{array}$ & $\begin{array}{c}43 \\
(23.8) \\
\end{array}$ & $\begin{array}{c}138 \\
(76.2)\end{array}$ & $\begin{array}{c}14 \\
(7.7) \\
\end{array}$ & $\begin{array}{c}167 \\
(92.3)\end{array}$ \\
\hline JDWNRH & $\begin{array}{c}136 \\
(80.0)\end{array}$ & $\begin{array}{c}34 \\
(20.0)\end{array}$ & $\begin{array}{c}141 \\
(82.9)\end{array}$ & $\begin{array}{c}29 \\
(17.2)\end{array}$ & $\begin{array}{c}134 \\
(78.8)\end{array}$ & $\begin{array}{c}36 \\
(21.2)\end{array}$ & $\begin{array}{c}43 \\
(25.4)\end{array}$ & $\begin{array}{c}126(74 . \\
6)\end{array}$ & $\begin{array}{c}26 \\
(15.3)\end{array}$ & $\begin{array}{c}144 \\
(84.7)\end{array}$ \\
\hline Overall & $\begin{array}{c}420 \\
(91.9)\end{array}$ & $\begin{array}{c}37 \\
(\mathbf{8 . 1})\end{array}$ & $\begin{array}{c}397 \\
(\mathbf{8 6 . 9})\end{array}$ & $\begin{array}{c}60 \\
(13.1)\end{array}$ & $\begin{array}{c}372 \\
(81.4)\end{array}$ & $\begin{array}{c}85 \\
(18.6)\end{array}$ & $\begin{array}{c}137 \\
(\mathbf{3 0 . 0})\end{array}$ & $\begin{array}{c}319 \\
(70.0)\end{array}$ & $\begin{array}{c}51 \\
(11.2)\end{array}$ & $\begin{array}{c}406 \\
(88.8)\end{array}$ \\
\hline
\end{tabular}

Note: Mean average number of days of illness =11.2 days (95\% CI 10.7, 11.7) (range 1-21)

Quantitative rapid slide agglutination Widal test was performed in 457 patients with suspected typhoid fever for presence or absence of antibodies against the $\mathrm{O}$ and $\mathrm{H}$ antigen of S. Typhi. Of these, $348(76 \%)$ were negative and $14(3.1 \%)$ patients showed the presence of both $\mathrm{O}$ antigen and $\mathrm{H}$ antigen. $56(12.3 \%)$ patients were positive for $\mathrm{O}$ antigen alone and 39 (8.5\%) showed presence of $\mathrm{H}$ antigen alone (Table 3 ).

Amongst those positive for $\mathrm{O}$ antigen, the commonest titre was 1:80 and 1:160 at 5.3\% $(n=24)$ each. Similarly, titre of 1:80 was the commonest reported dilution in patients who had a positive $\mathrm{H}$ titre (Table 4 ).

Table 3: Different titre of the two antigens tested

\begin{tabular}{lcccc}
\hline \multirow{2}{*}{ Titre } & \multicolumn{2}{c}{ O antigen } & \multicolumn{2}{c}{ H antigen } \\
\cline { 2 - 5 } & Frequency & $\begin{array}{c}\text { \% from total } \\
(\mathrm{n}=457)\end{array}$ & Frequency & $\begin{array}{c}\% \text { from total } \\
(\mathrm{n}=457)\end{array}$ \\
\hline $1: 80$ & 24 & 5.3 & 20 & 4.4 \\
$1: 160$ & 24 & 5.3 & 10 & 2.2 \\
$1: 320$ & 8 & 1.8 & 9 & 2.0 \\
\hline
\end{tabular}


There was no association of any of the symptoms to the antibody titres against $\mathrm{O}$ and $\mathrm{H}$ antigens (Table 5).

Table 4: Widal test results for different hospitals

\begin{tabular}{|c|c|c|c|c|}
\hline \multirow{3}{*}{ Hospital } & \multicolumn{4}{|c|}{ Widal test } \\
\hline & \multirow{2}{*}{$\frac{\text { Negative: titre }<1: 80}{(\%)}$} & \multicolumn{3}{|c|}{ Positive: titre $\geq 1: 80$} \\
\hline & & $\mathrm{H}$ antigen $(\%)$ & $\mathrm{O}$ antigen $(\%)$ & Mixed (\%) \\
\hline CRRH, Gelephu & $38(76.0)$ & $5(10.0)$ & $7(14.0)$ & $0(0.0)$ \\
\hline ERRH, Mongar & $35(62.5)$ & $9(16.1)$ & $12(21.4)$ & $0(0.0)$ \\
\hline PGH, Phuntsholing & $130(71.8)$ & $18(9.9)$ & $23(12.7)$ & $10(5.5)$ \\
\hline JDWNRH, Thimphu & $145(85.3)$ & $7(4.1)$ & $14(8.4)$ & $4(2.4)$ \\
\hline Total & $348(76.1)$ & $39(8.5)$ & $56(12.3)$ & $14(3.1)$ \\
\hline
\end{tabular}

Table 5: Association of symptoms to the two different antigens

\begin{tabular}{|c|c|c|c|c|c|c|}
\hline \multirow{3}{*}{ Symptoms } & \multicolumn{3}{|c|}{ Widal test } & \multirow{3}{*}{ Chi $^{2}$} & \multirow{3}{*}{$\begin{array}{c}\text { Degree } \\
\text { of } \\
\text { freedom }\end{array}$} & \multirow{3}{*}{$\begin{array}{c}\mathbf{P} \\
\text { value }\end{array}$} \\
\hline & \multirow{2}{*}{$\begin{array}{c}\text { Negative } \\
(<1: 80)\end{array}$} & \multicolumn{2}{|c|}{ Positive $(\geq 1: 80)$} & & & \\
\hline & & $O$ antigen & $H$ antigen & & & \\
\hline $\mathrm{Fe}$ & & $51(126)$ & $38(6.3)$ & 1.872 & 2 & 0.392 \\
\hline $\mathrm{Hea}$ & & & & 1 & 2 & 0.6 \\
\hline Bod & $2 \varepsilon$ & & & & 2 & 0.854 \\
\hline Diarı & 106 & $9.0)$ & $15(1$ & 3.3309 & 2 & 0.189 \\
\hline Constipation & $39(78.0)$ & $6(12.0)$ & $6(12.0)$ & 0.1122 & 2 & 0.945 \\
\hline
\end{tabular}

There seems to be three peaks during which suspected typhoid fever cases presented to the hospitals. Most cases reported to hospitals in August, followed by May and April. Very few cases were seen during January, the peak of winter. Patients' positive for O antigen reported to hospital throughout the year excepting the month of January. Most of these cases reported in the month of April followed by May and June. The patients who were positive for $\mathrm{H}$ antigen were reported throughout the year excepting the month of January and December (Figure 1).

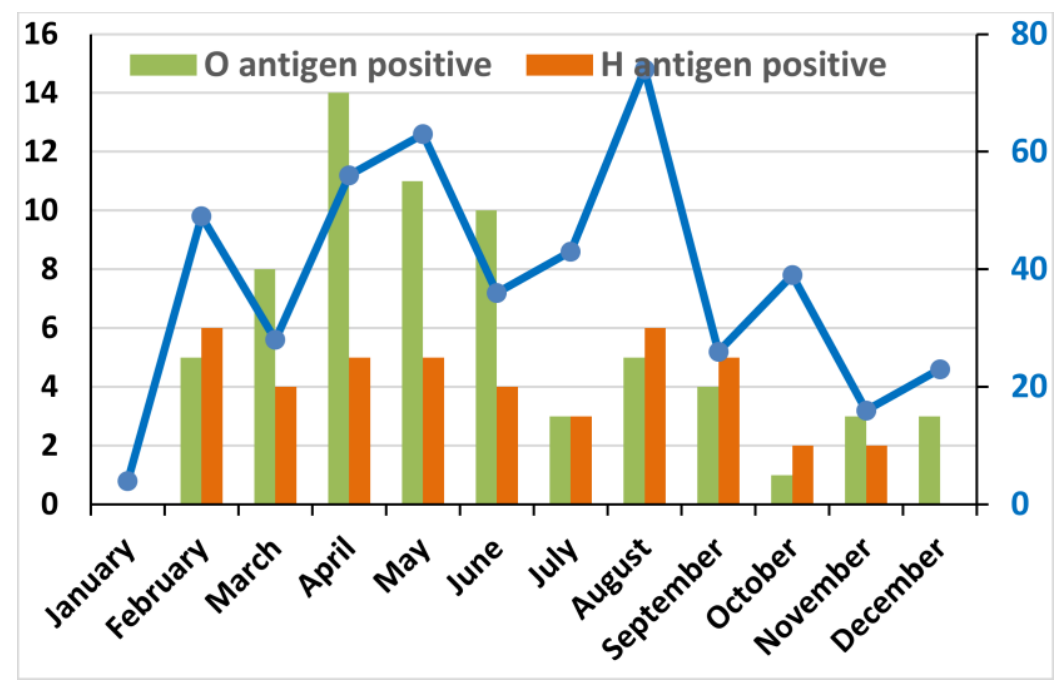

Figure 1: Frequency graph with the cases in different months 
More than $97 \%(\mathrm{n}=447)$ of blood cultures were sterile, and $S$. Typhi was isolated only in $1.1 \%(\mathrm{n}=5)$, all of which were from the JDWNRH.

Of the 5 isolates of $S$. Typhi, all were susceptible to the commonly used antibiotics (chloramphenicol, ciprofloxacin, gentamicin and ceftriaxone). Only one isolate showed resistance to both amoxicillin and nalidixic acid. There was one isolate of $S$. Paratyphi A which was susceptible to all antibiotics. Only two (33\%) of the six blood culture positive cases had positive Widal titres. Other isolates from blood culture included Escherichia coli $(\mathrm{n}=1)$, Staphylococcus aureus $(\mathrm{n}=1)$ and coagulase negative Staphylococcus $(\mathrm{n}=2)$.

In our study, if blood culture positivity was considered the gold standard true indicator of typhoid fever, the case incidence of typhoid fever was only $1.3 \%$ but the incidence increased to $23.9 \%$ using the Widal test as the diagnostic indicator (titre $\geq 1: 80$ ). However, when blood culture positivity was considered the true indicator of typhoid fever, Widal test showed a sensitivity of only $33.3 \%$, specificity of $76.3 \%$ with positive predictive value (PPV) of $1.8 \%$ and negative predictive value (NPPV) of $98.8 \%$.

\section{Discussion}

Overall, there has been a decline in morbidity and mortality due to infectious diseases in Bhutan, probably associated with improved public education, hygiene and sanitation and prevention and control measures. ${ }^{4}$ The total number of patients enrolled in this study may not necessarily reflect the disease burden in the study sites since patient enrolment has to do a lot with the prescription pattern of the doctors and pro-activeness of the laboratory staff. This was obvious from the variation in number of cases among the hospitals. The equal number of both sexes in the study reflects that both sexes are equally exposed and prone to the food and water borne infections like typhoid fever.

The mean duration of illness of 11.2 days before seeking medical consultation was longer than expected and worrying since complications like severe sepsis, gastrointestinal perforation and salmonella meningitis can ensue in true cases of typhoid if not treated promptly. This finding in general indicates the need for public education on health care seeking behaviour during illnesses in general and during febrile illnesses in particular. This is important since traditional beliefs and local remedy seeking behaviour before medical consultations is still prevalent despite recent improvements.

The generally accepted poor sensitivity and specificity of the Widal test has been affirmed in the Bhutanese setting as well since there was no association with patient symptoms and positive blood culture. The sensitivity of $33.3 \%$ and specificity of $76.3 \%$ with positive predictive value (PPV) of $1.8 \%$ was lower than a study in paediatric population in India but the negative predictive value (NPPV) of $98.8 \%$ was higher ${ }^{5}$. This is an important finding as the Ministry of Health, Bhutan is considering replacing the Widal test with other supposedly more sensitive and specific tests like Typhidot ${ }^{6}$, a dot Enzyme-linked immunosorbent assay (ELISA) kit that detects IgM and IgG antibodies against the outer membrane protein (OMP) of the Salmonella Typhi. Also, in an endemic country with unknown baseline antibody titres, the Widal test is more likely to give false positive results ${ }^{7}$, and this seems to be happening in Bhutan. However, in the absence of an alternative, the Widal test, especially with high titres, is useful and specific in about $74 \%$ of true typhoid fever cases if interpreted properly. ${ }^{8}$ In an Indian study, at a cut off $\geq 1: 50$ for $\mathrm{O}$ agglutinins or $\geq 1: 100$ for $\mathrm{H}$ agglutinins, the Widal test 
gave a sensitivity of $71.43 \%$ and specificity of only $47.31 \%$. A low positive predictive value of $9.25 \%$ and a negative predictive value of $95.65 \%$ was noted making the test unreliable in an endemic setting. ${ }^{5}$

The seasonal variation of the cases is probably related to favourable transmission in the monsoon summer and this finding agrees with a study in a densely populated area in Katmandu, Nepal ${ }^{9}$, a country with similar geographic setting to Bhutan. The case incidence of typhoid fever of $1.3 \%$ (6/457) (by blood culture positives) was lower than expected for a probably endemic country. The case incidence however increased to $23.9 \%$ if Widal test was used as the diagnostic indicator. The culture positivity rate of $2.2 \%$, of which only $1.3 \%$ (6 isolates) were Salmonella was very low. This could have resulted from poor case inclusion criteria for typhoid fever cases, unsatisfactory blood samples (single sample) and culture techniques in the laboratories and also due to prior antibiotic treatment of patients. The high sensitivity against commonly used antibiotics, despite fewer isolates, is quite encouraging and useful for the clinical field staff since almost all patients are treated routinely without microbiological laboratory support. However, if not intervened appropriately sooner, the repeated testing and positive results with Widal tests, leading to antibiotic treatment and retreatment of the patients can contribute to the rising problem of antibiotic resistance in the community.

\section{Conclusion}

From this study, it was apparent that the traditional Widal test does not correspond well with the symptoms. Typhoid fever is not properly diagnosed with currently available laboratory tests but over-diagnosed and treated clinically. Therefore, it is timely to initiate more sensitive and specific diagnostic tests. There is a need to conduct a proper study to establish the baseline antibody titre in the general population, find the actual burden of the disease and strengthen laboratory practices in the country for detection of common febrile illness. Till such a time, the Widal test needs to be used cautiously with definite cut-off titres.

\section{Limitations}

This study has some pertinent limitations. The work was carried out in a country with no baseline titre for Widal tests interpretation, there is limited laboratory capacity and the cut-off titres in clinical practice were not uniformly interpreted. The patients were enrolled as typhoid fever 'suspects', only with clinical exclusion of any other obvious febrile illnesses and other causes of febrile illnesses (pyrexia of unknown origin) like malaria, leptospirosis, brucellosis, dengue fever etc. that can present with the same symptoms were not tested. Very few clinical symptoms have been considered and noted in the questionnaire making detailed correlation with laboratory results difficult.

\section{Conflict of interest}

The authors declare that they have no competing interest.

\section{Acknowledgement}

The authors would like to acknowledge the funding received from the World Health Organization Country Office, Bhutan, through the Department of Public Health, Ministry of Health, Bhutan for study protocol development meetings and briefing of the laboratory and clinical staffs involved in the surveillance. 


\section{Authors' contribution}

All authors contributed equally and the final manuscript has been reviewed and approved by all.

\section{References}

1. Crump JA, Luby SP, Mintz ED. The global burden of typhoid fever. Bulletin of the World Health Organization. 2004;82(5):346-353. https://www.ncbi.nlm.nih.gov/pmc/articles/PMC2622843/

2. Ochiai RL, Acosta CJ, Danovaro-Holliday M, et al. A study of typhoid fever in five Asian countries: disease burden and implications for controls. Bulletin of the World Health Organization. 2008; 86(4):260-268. doi : https://dx.doi.org/10.2471\%2FBLT.06.039818

3. Steinberg E, Bishop R, Haber P, et al. Typhoid fever in travelers: who should be targeted for prevention? Clinical infectious diseases. 2004; 39(2):186-191. doi : https://dx.doi.org/10.1086/421945

Annual Health Bulletin 2011: Ministry of Health, Royal Government of Bhutan; 2011. Accessed on http://www.health.gov.bt/wp-content/uploads/ftps/annual-healthbulletins/Annual\%20Health\%20Bulletin-2011/ahbContent2011.pdf

4. Lalremruata R, Chadha S, Bhalla P. Retrospective Audit of the Widal Test for Diagnosis of Typhoid Fever in Pediatric Patients in an Endemic Region. Journal of clinical and diagnostic research: JCDR. 2014; 8(5):DC22. https://dx.doi.org/10.7860/JCDR/2014/7819.4373

5. Sherwal B, Dhamija R, Randhawa V, et al.. A comparative study of Typhidot $\mathrm{M}$ and widal test in patients of Typhoid fever. Journal of Indian Academy of Clinical Medicine. 2004; 5(3).244-6. http://medind.nic.in/jac/t04/i3/jact04i3p244.pdf

6. Kulkarni M, Rego S. Value of single Widal test in the diagnosis of typhoid fever. Indian pediatrics. 1994; 31(11):1373-1377. http://indianpediatrics.net/nov1994/1373.pdf

7. Parry CM, Hoa NTT, Diep TS, et al. Value of a single-tube Widal test in diagnosis of typhoid fever in Vietnam. Journal of clinical microbiology. 1999; 37(9):2882-2886. https://www.ncbi.nlm.nih.gov/pmc/articles/PMC85403/

8. Karkey A, Arjyal A, Anders KL, et al. The burden and characteristics of enteric fever at a healthcare facility in a densely populated area of Kathmandu. PloS one. 2010; 5(11):e13988. doi : https://dx.doi.org/10.1371/journal.pone.0013988 